\title{
Impact of Insulin Degludec in Type 2 Diabetes: Real-World Data on Effectiveness and Safety
}

\author{
Paola Ponzani - Cesare Berra • Alessandra Di Lelio - Paola Del Sindaco • \\ Chiara Di Loreto · Francesco Reggiani · Giuseppe Lucisano • \\ Maria Chiara Rossi (D)
}

Received: August 3, 2018 / Published online: September 21, 2018

(c) The Author(s) 2018

\begin{abstract}
Introduction: Real-world evidence on effectiveness and safety of insulin degludec (IDeg) in patients with diabetes is a priority. The aim of the study was to evaluate patterns of use and the long-term effectiveness and safety of IDeg in routine clinical practice.

Methods: This was an observational longitudinal study. A retrospective chart review of all patients with type 2 diabetes treated with IDeg was performed and temporal trends in clinical outcomes were assessed. All data was stratified
\end{abstract}

Enhanced digital features To view enhanced digital features for this article go to https://doi.org/10.6084/ m9.figshare.7059281.

Electronic supplementary material The online version of this article (https://doi.org/10.1007/s13300018-0511-4) contains supplementary material, which is available to authorized users.

P. Ponzani

SSD Endocrinologia, Diabetologia e Malattie

Metaboliche ASL3 Genovese, Genoa, Italy

C. Berra · F. Reggiani

Humanitas Research Institute, Rozzano, MI, Italy

A. Di Lelio · G. Lucisano - M. C. Rossi ( $₫)$

CORESEARCH, Center for Outcomes Research and Clinical Epidemiology, Pescara, Italy

e-mail: rossi@coresearch.it

P. Del Sindaco · C. Di Loreto

Servizio di Diabetologia del Perugino USL Umbria 1, Perugia, Italy by treatment modality: the switch group consisted of patients already treated with another basal insulin before initiating IDeg; the add-on group consisted of basal insulin-naïve patients. Results: Overall, 247 patients were analyzed (55 in the add-on group and 192 in the switch group), mean age $67.0 \pm 10.9$ years , and diabetes duration $16.3 \pm 8.9$ years. Median (interquartile range) follow-up was 9.7 (8.0-11.9) months. In the add-on group, improvements were found in glycated hemoglobin (HbA1c) $(-1.68 \% ; p<0.0001)$, fasting blood glucose (FBG) $(-64.7 \mathrm{mg} / \mathrm{dL} ; p<0.0001)$, post-prandial glucose (PPG) $(-81.1 \mathrm{mg} / \mathrm{dl} ; p<0.0001)$, and glycemic variability (i.e., standard deviation of blood glucose) $(-11.6 \mathrm{mg} / \mathrm{dl} ; p=0.04)$. Even in the switch group, improvements were found in HbA1c $(-0.57 \%$; $p<0.0001)$, FBG $(-28.1 \mathrm{mg} /$ $\mathrm{dL} ; \quad p<0.0001)$, and PPG $(-22.6 \mathrm{mg} / \mathrm{dl}$; $p=0.001)$. Body weight increase during the follow-up was not statistically significant vs. baseline in both groups. Benefits on overall, nocturnal, and severe hypoglycemia were found in the switch group.

Conclusion: These real-world data documented that initiating IDeg or switching to IDeg from other basal insulins in type 2 diabetes was associated with significant improvement in metabolic control without significant weight gain; a decrease in the risk of hypoglycemia was observed when switching to IDeg from another basal insulin. 
Keywords: Add-on; Effectiveness; Glycemic variability; Hypoglycemia; Insulin degludec; Real-world data; Switch; Type 2 diabetes

\section{INTRODUCTION}

Ultra-long-acting insulin degludec (IDeg) is a recent therapeutic option for patients with diabetes requiring treatment with basal insulin [1]. IDeg has been extensively tested in the BEGIN $^{\circledR}$ studies, a comprehensive research program involving a wide range of patients with diabetes [2-6]. These trials consistently showed that IDeg reduced glycated hemoglobin (HbA1c, primary outcome) by over $1 \%$ similarly to glargine; furthermore, a lower risk of hypoglycemia, especially nocturnal episodes, was documented. The lower day-to-day variability of IDeg is responsible for the more predictable effect and the more favorable safety profile of IDeg vs. glargine $[7,8]$. These findings suggest that IDeg represents an advance in diabetes therapy owing to the positive impact on hypoglycemia and fear of hypoglycemia, recognized as main barriers to the achievement of glucose targets and patient quality of life $[9,10]$.

Furthermore, additional benefits on fasting plasma glucose (FPG) were obtained with IDeg vs. glargine in insulin-naïve adults with type 2 diabetes (T2DM) [3, 4, 11], while comparable decreases in FPG were obtained in patients treated with basal-bolus regimens [2].

Finally, weight gain was an issue in these trials, since it represents another key barrier in patients treated with insulin [12]. As expected, a small increase in weight gain (ranging from 2 to $4 \mathrm{~kg}$ ) was found both in insulin-naïve and in basal-bolus patients during the follow-up, without statistically significant differences between IDeg and glargine [2-4].

In addition to experimental studies, realworld data are strongly required to obtain an overall picture of the effectiveness and safety of any new drug when used under routine clinical practice conditions $[13,14]$. Therefore, it is important to assess the impact of IDeg on metabolic control, hypoglycemia, and body weight when adopted in real-world populations. However, real-world data on the impact of IDeg are still rare at the moment.

The overall aim of the study was to evaluate patterns of use and the long-term effectiveness and safety of IDeg in routine clinical practice. The primary objective of the study was to evaluate whether the reduction in HbA1c observed in clinical trials could be attained in patients with T2D routinely managed in outpatient clinics. Secondary objectives included evaluation of the attainment of beneficial effects on blood glucose levels and variability, body weight, and hypoglycemia under the same conditions.

\section{METHODS}

This was an observational, retrospective cohort study based on T2DM patients consecutively attending three diabetes outpatient clinics in Italy.

Two different cohorts were identified: the switch group consisted of patients already treated with another basal insulin before initiating IDeg; the add-on group consisted of basal insulin-naïve patients.

To minimize the selection bias, all consecutive patients who had started the treatment with IDeg (insulin-naïve subjects or switched to IDeg from another basal insulin) under routine clinical practice conditions were included in the study.

Baseline (i.e., IDeg start date, T0) patient characteristics included age, sex, body mass index (BMI), diabetes duration, glucose-lowering treatment, and diabetes-related complications. Chronic kidney disease was defined as an estimated glomerular filtration rate below $60 \mathrm{ml} / \mathrm{min}$ (CKD-EPI formula).

Data on glycated hemoglobin (HbA1c), fasting blood glucose (FBG), post-prandial glucose (PPG), standard deviation of mean blood glucose (glycemic variability), body weight, doses of basal and short-acting insulin, and overall, nocturnal, and severe hypoglycemia episodes was collected at baseline visit and during the two subsequent follow-up visits, scheduled according to the routine clinical practice (T1 and $\mathrm{T} 2$ ). 
HbA1c levels were measured in the hospital laboratories, all adopting standardized methods (DCCT-aligned HbA1c method).

Data was acquired from electronic medical records.

Downloaded blood glucose data from glucose meters routinely used by the patients providing data on FBG, PPG, glycemic variability, and hypoglycemia included all the values recorded during the last 3 months before each visit. Overall hypoglycemia was defined as all blood glucose values less than $70 \mathrm{mg} / \mathrm{dl}$, severe hypoglycemia as a hypoglycemia episode requiring assistance by a third person. Nocturnal hypoglycemia was defined as blood glucose value less than $70 \mathrm{mg} / \mathrm{dl}$ recorded between midnight and 7.00 a.m.

The study protocol was approved by local ethics committees and all patients signed the informed consent. All procedures followed were in accordance with the ethical standards of the responsible committee on human experimentation (institutional and national) and with the Helsinki Declaration of 1964, as revised in 2013.

\section{Statistical Analysis}

All data was stratified by treatment type (switch group; add-on group).

Descriptive data was expressed as mean \pm standard deviation (SD) for continuous variables, and percentage for categorical variables.

Longitudinal linear models for repeated measures were applied to assess trends over time in continuous endpoints (HbA1c, FBG, PPG, glycemic variability, weight, insulin dose). All longitudinal models take into consideration three time points, i.e., T0, T1, and T2. Only for insulin doses, an additional time point was considered $(\mathrm{T}-1$, corresponding to the last prescribed dose of the previous basal insulin before the initiation of IDeg). For all longitudinal models, an unstructured correlation type was used to account for within-patient correlation over time and unequal follow-up. Results were expressed as estimated mean and estimated mean change from baseline with their 95\% confidence intervals (CIs), and $p$ values less than 0.05 were considered statistically significant.

Proportions of patients with at least one hypoglycemic episode were assessed at three time points, i.e., during 3 months before IDeg start date (T0), during 3 months before the first follow-up visit (T1), and during 3 months before the second follow-up visit (T2).

All statistical analyses were performed with SAS software release 9.4 (SAS Institute, Cary, NC, USA).

\section{RESULTS}

Data on 247 patients with T2DM who started the treatment with IDeg between November 2014 and November 2015 was analyzed. The first follow-up visit (T1) was performed after (median and interquartile range) 4.6 (3.4-6.1) months from baseline (T0), while the second follow-up visit (T2) was performed after 9.7 (8.0-11.9) months from T0.

Information on data completeness at each visit is in Table $\mathrm{S} 1$.

Overall, 55 patients started IDeg as basal insulin-naïve patients and 192 patients switched to IDeg from another basal insulin. Baseline characteristics overall and by treatment modality are reported in Table 1 . The mean age was $67.0 \pm 10.9$ years, men represented $50.6 \%$ of the sample, and mean diabetes duration was $16.3 \pm 8.9$ years. At baseline, mean BMI was $31.1 \pm 6.1 \mathrm{~kg} / \mathrm{m}^{2}$, chronic kidney disease was present in $22.7 \%$ of the cases, whereas $25.5 \%$ of patients had a history of cardiovascular events. Patients in the switch and add-on groups differed in terms of diabetes duration $(17.7 \pm 8.8$ vs. $11.1 \pm 7.4$ years; $p<0.0001)$ and concomitant glucose-lowering treatments, especially short-acting insulin $(81.3 \%$ vs. $10.9 \%$; $p<0.0001$ ) (Table 1$)$. In the add-on group: $83.6 \%$ self-administered 1 insulin injection/day, $5.5 \%$ self-administered 3 insulin injections/day, and $10.9 \%$ self-administered 4 insulin injections/day. In the switch group, 19.3, 1.6, 8.3, and $70.8 \%$ self-administered $1,2,3$, and 4 insulin injections/day, respectively.

In the add-on group, relevant improvements were found in HbA1c $(-1.68 \% ; p<0.0001)$, 
Table 1 Baseline patient characteristics

\begin{tabular}{|c|c|c|c|c|}
\hline Variables & Overall & Add-on & Switch & $p$ value* $^{*}$ \\
\hline No. group & 247 & 55 & 192 & \\
\hline Age (years) & $67.0 \pm 10.9$ & $67.1 \pm 11.3$ & $66.9 \pm 10.9$ & 0.94 \\
\hline \multicolumn{5}{|l|}{$\operatorname{Sex}(\%)$} \\
\hline Male & 50.6 & 43.6 & 52.6 & 0.24 \\
\hline Female & 49.4 & 56.4 & 47.4 & \\
\hline Diabetes duration (years) & $16.3 \pm 8.9$ & $11.1 \pm 7.4$ & $17.7 \pm 8.8$ & $<0.0001$ \\
\hline $\operatorname{BMI}\left(\mathrm{kg} / \mathrm{m}^{2}\right)$ & $31.1 \pm 6.1$ & $29.6 \pm 5.3$ & $31.5 \pm 6.3$ & 0.11 \\
\hline Chronic kidney disease (\%) & 22.7 & 20.0 & 23.4 & 0.59 \\
\hline Previous CV event (\%) & 25.5 & 20.0 & 27.1 & 0.29 \\
\hline Previous treatment with basal insulin other than degludec (\%) & 77.7 & 0 & 100 & - \\
\hline \multicolumn{5}{|l|}{ Other glucose-lowering treatments } \\
\hline Metformin & 26.1 & 48.1 & 24.0 & 0.0007 \\
\hline Sulfonylurea & 16.8 & 32.7 & 12.5 & 0.0006 \\
\hline Pioglitazone & 2.5 & 3.8 & 2.1 & 0.47 \\
\hline Incretin mimetics & 11.9 & 30.8 & 6.8 & $<0.0001$ \\
\hline Sodium/glucose cotransporter 2 & 0.4 & 0 & 0.5 & 0.60 \\
\hline Short-acting insulin & 65.6 & 10.9 & 81.3 & $<0.0001$ \\
\hline
\end{tabular}

Data are mean and standard deviation or proportions

${ }^{*}$ Statistically significant differences $(p<0.05)$ are in bold text

FBG $\quad(-64.7 \mathrm{mg} / \mathrm{dL} ; \quad p<0.0001), \quad$ and PPG $(-81.1 \mathrm{mg} / \mathrm{dl} ; p<0.0001)$. In the switch group, improvements were found in HbA1c $(-0.57 \%$; $p<0.0001)$, FBG $(-28.1 \mathrm{mg} / \mathrm{dL} ; p<0.0001)$, and PPG $(-22.6 \mathrm{mg} / \mathrm{dl} ; p=0.001)$. A not statistically significant decrease in glycemic variability was found $(-3.7 \mathrm{mg} / \mathrm{dl} ; p=0.06)$. Average body weight increase during the followup $(+1.41 \mathrm{~kg}$ in the add-on group and $+0.52 \mathrm{~kg}$ in the switch group) was not statistically significant vs. baseline (Table 2 and Fig. S1).

Changes in IDeg and short-acting insulin doses at each visit were assessed (Fig. 1). In the switch group, the dose of the previous basal insulin was also tested in the model (Fig. 1). At $\mathrm{T}-1,45.3 \%$ of the patients in the switch group were treated with detemir once daily, $5.7 \%$ with detemir twice daily, $43.8 \%$ with glargine once daily, $3.6 \%$ with glargine twice daily, and $1.6 \%$ with neutral protamine Hagedorn insulin. In the switch group, a reduction in HbA1c levels was obtained despite the total insulin dose being almost unmodified, with a slight increase in the dose of basal insulin and a slight decrease in short-acting insulin dose. In the add-on group, the dose of basal insulin was progressively titrated from $\mathrm{T} 0$ to $\mathrm{T} 2$, reaching an average dose of $20.6 \mathrm{IU}$ at T2. At the end of the study (T2), the mean dose of IDeg was $0.24 \mathrm{IU} / \mathrm{kg}$ in the add-on group and $0.33 \mathrm{IU} / \mathrm{kg}$ in the switch group.

As for hypoglycemic episodes, in the switch group, overall, nocturnal, and severe hypoglycemia became less frequent after IDeg initiation vs. previous basal insulin and no severe 


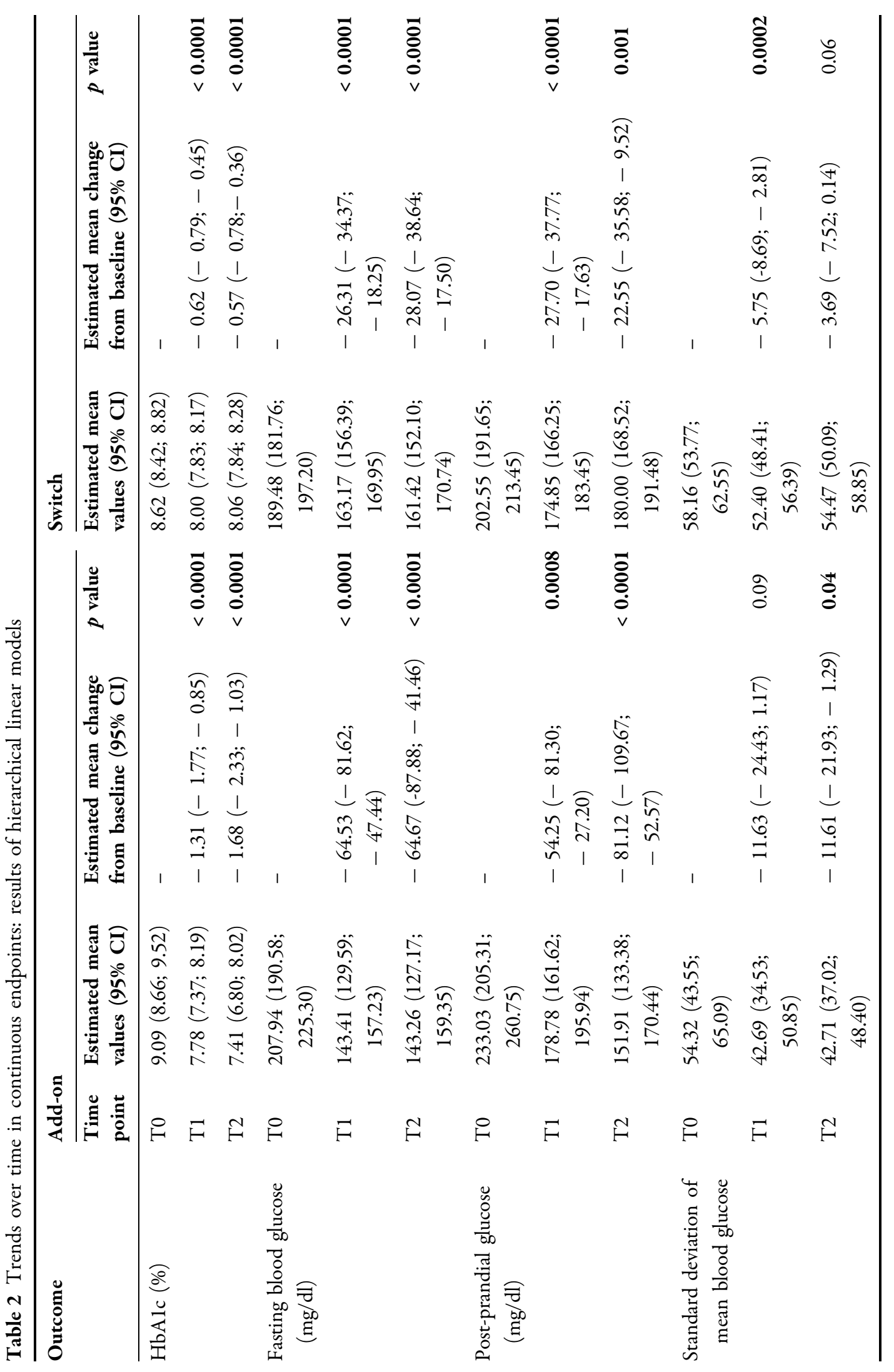




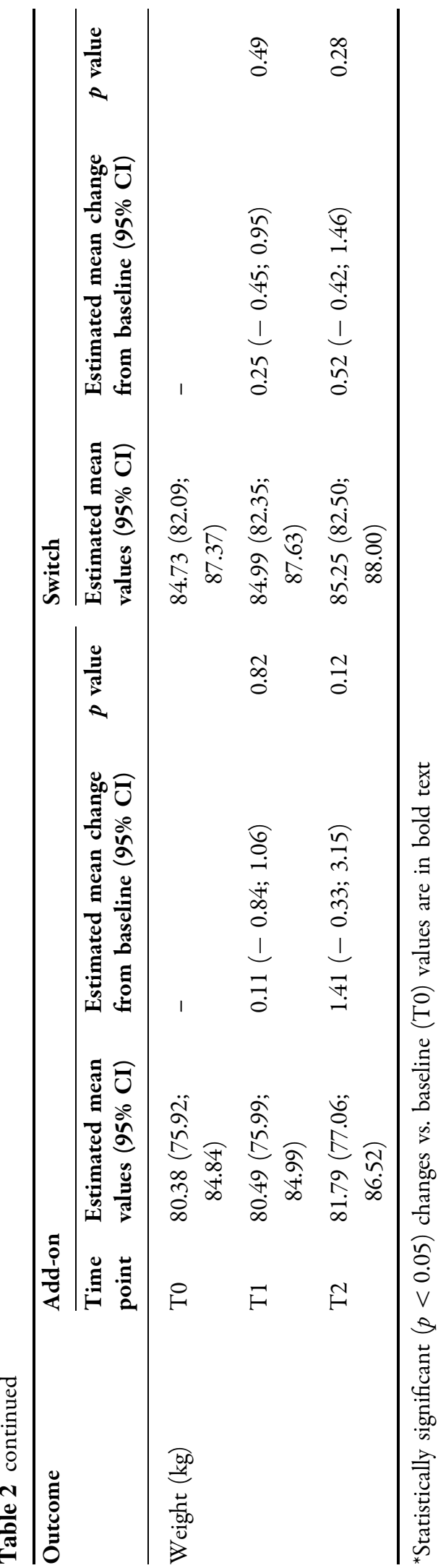

episode occurred after IDeg initiation (Fig. 2). In the add-on group, small proportions of patients experienced overall hypoglycemia during the study, while no severe and nocturnal episodes occurred (Fig. 2).

\section{DISCUSSION}

Our study shows the impact of initiating IDeg insulin therapy in the real world, outside the rigid schemes of randomized clinical trials. The decision to start IDeg as an add-on to the current therapy or as a substitute for another basal insulin therapy was taken by the diabetologists on clinical grounds, i.e., because of poor metabolic control or high risk of hypoglycemia.

Consistent with the BEGIN studies on T2DM [2-6], our study confirms that IDeg provides statistically significant and clinically meaningful improvements in HbA1c. In the BEGIN studies, HbA1c was improved by $1.1-1.3 \%$ in insulin-naïve and in basal-bolus patients. In our study, on the basis of routine data, HbA1c was reduced by $1.61 \%$ in the add-on group and $0.52 \%$ in the switch group. Among the latter, $60 \%$ used basal insulin in association with short-acting insulin, while in the BEGIN study [6] all switching patients were on basal-bolus regimens. It is important to underline that in the switch group the positive effects on metabolic control were obtained despite the total insulin dose being almost unmodified, thus suggesting that the benefits should be attributed to IDeg per se, rather than to an optimization of insulin doses.

In our study, FBG, PPG, glycemic variability, and frequency of hypoglycemia also improved from baseline to the end of the follow-up both in add-on and switch groups.

Interestingly, in our real-world cohort, a lower increase in body weight was found in comparison with the BEGIN studies in both subgroups, probably as a result of the need for lower doses of IDeg in our study (0.2-0.3 IU/kg) as compared to those utilized in the BEGIN trials (about 0.5-0.6 IU/kg). Presumably, the lower doses were justified by lower BMI of this population and by the treat-to-target design of the BEGIN studies. 

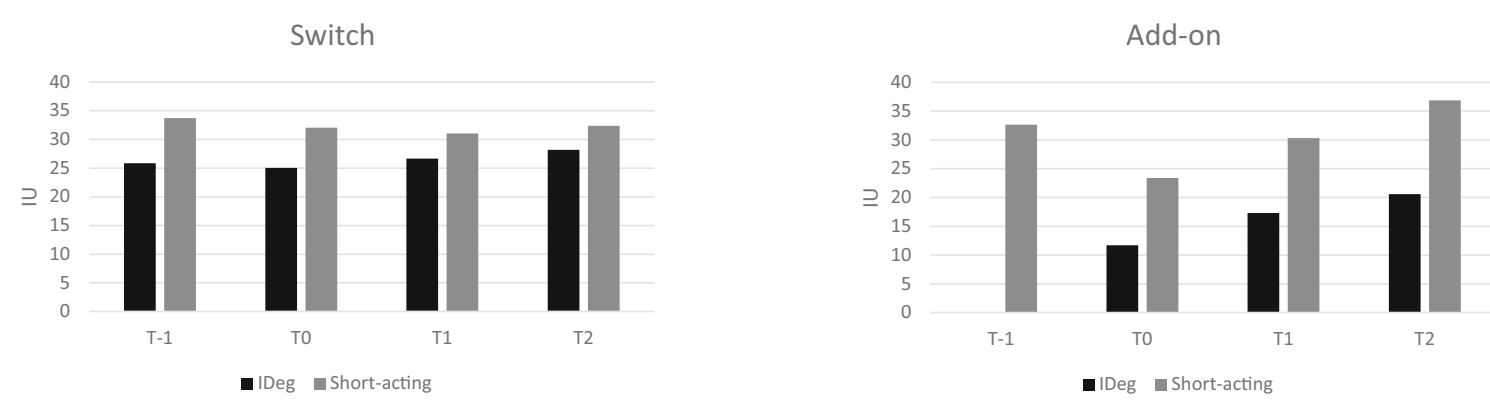

\begin{tabular}{|c|c|c|c|c|c|c|c|c|}
\hline & \multicolumn{4}{|c|}{ Switch } & \multicolumn{4}{|c|}{ Add-on } \\
\hline & Time-point & $\begin{array}{l}\text { Estimated mean } \\
\text { values }(95 \% \mathrm{Cl})\end{array}$ & $\begin{array}{l}\text { Estimated mean change } \\
\text { from } \mathrm{RC}(95 \% \mathrm{Cl})\end{array}$ & $p$-value & Time-point & $\begin{array}{l}\text { Estimated mean } \\
\text { values }(95 \% \mathrm{Cl})\end{array}$ & $\begin{array}{l}\text { Estimated mean change } \\
\text { from } \mathrm{RC}(95 \% \mathrm{Cl})\end{array}$ & $p$-value \\
\hline \multirow[t]{4}{*}{ Basal insulin } & $\mathrm{T}-1(\mathrm{RC})^{*}$ & $25.86(23.83-27.89)$ & - & & - & - & - & - \\
\hline & T0 & $25.06(23.25-26.87)$ & $-0.80(-1.63 ; 0.03)$ & 0.06 & T0 (RC) & $11.71(10.75-12.67)$ & - & \\
\hline & T1 & $26.66(24.73-28.59)$ & $+0.80(-0.26 ; 1.86)$ & 0.14 & $\mathrm{~T} 1$ & $17.33(14.95-19.71)$ & $+5.62(3.39 ; 7.85)$ & $<0.0001$ \\
\hline & $\mathrm{T} 2$ & $28.17(26.02-30.32)$ & $+2.31(0.99 ; 3.63)$ & 0.0007 & $\mathrm{~T} 2$ & $20.60(16.83-24.37)$ & $+8.89(5.21 ; 12.57)$ & $<0.0001$ \\
\hline \multirow[t]{4}{*}{ Short-acting insulin } & $\mathrm{T}-1$ & $33.73(31.00-36.46)$ & & & $T-1$ & $32.65(24.75-40.55)$ & & \\
\hline & T0 & $32.03(29.40-34.66)$ & $-1.71(-2.97 ;-0.45)$ & 0.009 & TO & $23.38(16.09-30.67)$ & $-9.28(-15.60 ;-2.96)$ & 0.01 \\
\hline & $\mathrm{T} 1$ & $31.02(28.12-33.92)$ & $-2.72(-4.26 ;-1.18)$ & 0.0007 & $\mathrm{~T} 1$ & $30.32(24.28-36.36)$ & $-2.34(-11.53 ; 6.85)$ & 0.63 \\
\hline & $\mathrm{T} 2$ & $32.37(29.27-35.47)$ & $-1.36(-3.22 ; 0.50)$ & 0.15 & $\mathrm{~T} 2$ & 36.88 (28.39-45.37) & $4.23(-9.03 ; 17.49)$ & 0.54 \\
\hline
\end{tabular}

$\mathrm{T}-1$ refers to the last prescribed dose of the previous basal insulin before degludec initiation; $\mathrm{RC}=$ reference class; *Mean dose of the previous basal insulin. ** Data refers to 154 patients in the Switch group and 6 patients in the Add-on group

Fig. 1 Changes in basal and short-acting insulin dose (IU) during the study
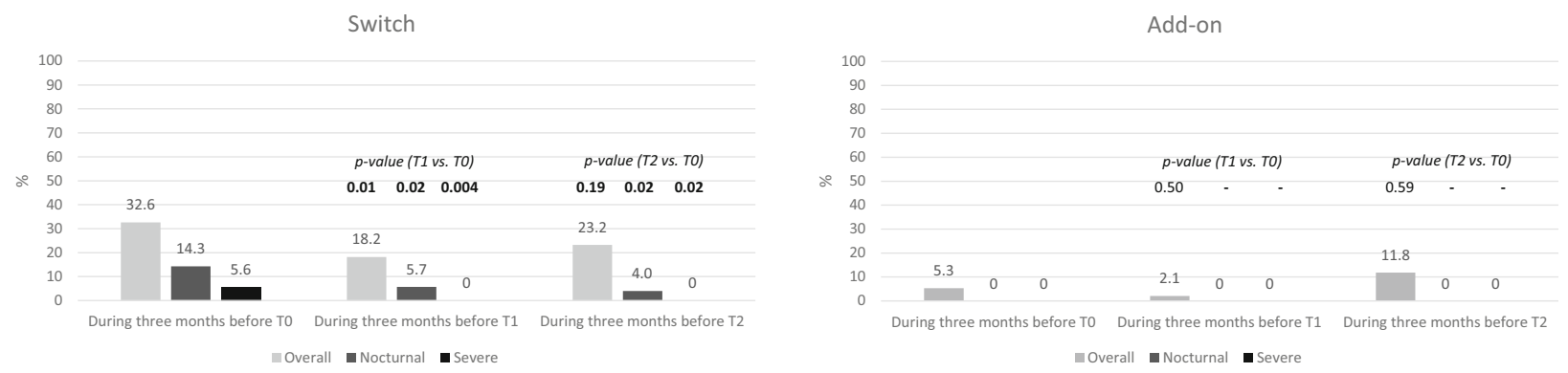

Fig. 2 Hypoglycemia: proportion of patients with at least one episode during 3 months before each study visit

Our data may also be compared with findings from other observational studies. In a European multicenter, retrospective chart review study on 822 cases with T2DM $(22.4 \%$ on basal insulin-only regimens and $74.5 \%$ on basal-bolus regimens) after 12 months from IDeg start, HbA1c decreased by $5.7 \mathrm{mmol} / \mathrm{mol}$ $(p<0.001)$, FBG decreased by $1.47 \mathrm{mmol} / \mathrm{L}$ $(p<0.001)$, rates of overall and nocturnal nonsevere hypoglycemia were $60 \%$ and $90 \%$ lower than in the previous period, and no weight change occurred [15]. Data from the DPV registry on 61 T2DM patients showed that IDeg was associated with a decrease in $\mathrm{HbA1c}$ from $7.9 \%$ to $7.1 \%$, without change in BMI and insulin doses after 3-15 months [16]. In a Japanese study on 135 cases with T2DM, HbA1c decreased by $0.3 \%$ after 12 months from the switch to IDeg from another basal insulin, without significant change in body weight and with a reduction in the frequency of nocturnal hypoglycemic episodes [17]. In a study conducted in Israel and involving 211 patients with T2DM, switching from another insulin to IDeg significantly improved glycemic control without significant weight gain and with only a modest increase in insulin dose after IDeg initiation [18]. Recently, a comparative effectiveness analysis of IDeg vs. glargine 300 (DELIVER D+ cohort study) showed that mean HbA1c reductions among patients were similar in the two cohorts, consistent with our results [19]. Finally, the safety of IDeg was documented in a 
study on hospitalized, mainly elderly patients [20].

As a major strength, our study provides an overall picture on effectiveness and safety of IDeg in two clinically different populations, i.e., insulin-naïve and switching patients. Other strengths are the multicenter nature of the study, the sample size, the duration of the follow-up compared to the available observational studies, the quality of data despite their routine care nature, and the comprehensive set of endpoints. Among the limitations, the lack of a comparator arm should be mentioned. Furthermore, possible modifications in type or doses of the concomitant glucose-lowering treatments may represent confounders; however, investigators reported no major modifications in the concomitant treatments during the follow-up. Some of the analyses focusing on the add-on group could be limited by the small sample size, precluding the possibility of showing statistically significant differences. No information about the type of short-acting and previous basal insulin formulations was collected. Participants probably performed a different number of blood glucose measurements according to the different glucose-lowering drugs they took; this could give heterogeneous estimates because some patients could have a very low, or conversely a very high, number of measurements.

\section{CONCLUSIONS}

This real-world study demonstrated that initiating IDeg or switching patients with T2DM to IDeg from other basal insulins significantly improves glycemic control, reduces glycemic variability, and decreases the risk of hypoglycemia, without significant weight gain.

\section{ACKNOWLEDGEMENTS}

Funding. This was a non-profit investigatorinitiated initiative. No funding or sponsorship was received for the study itself. The authors of the publication are fully responsible for its contents and conclusions.

Medical Writing and Editorial Assistance. The agency Airon Communication supported the clinicians with editorial assistance. This assistance included statistical analysis and medical writing services (provided by CORESEARCH - www.coresearch.it) and article processing charges. These services were funded through an unconditional grant from Novo Nordisk S.p.A. The authors of the publication are fully responsible for its contents and conclusions. Novo Nordisk S.p.A. did not influence and has not been involved in the data interpretation and statistical analysis presented in the manuscript. All authors had full access to all of the data in this study and take complete responsibility for the integrity of the data and accuracy of the data analysis.

Authorship. All named authors meet the International Committee of Medical Journal Editors (ICMJE) criteria for authorship for this manuscript, take responsibility for the integrity of the work as a whole, and have given final approval to the version to be published.

Disclosures. Paola Ponzani: Novo Nordisk, GSK, Jansen, Bayer and Novartis for research grants, Eli Lilly for honoraria for speaking and position on advisory board; Cesare Berra: consulting fees from Novo Nordisk, Lilly, Boehringer Ingelheim, Sanofi, Johnson \& Johnson and Bayer; research support from AstraZeneca and Takeda; member of advisory board for Lilly, Novo Nordisk, Boehringer Ingelheim, Sanofi and AstraZeneca; investigator in clinical trials sponsored by Lilly, Sanofi and Novo Nordisk; Chiara Di Loreto: research support from Roche diagnostics; Maria Chiara Rossi: research support from ForaCare, Artsana, Sanofi, Novo Nordisk, Dexcom, Medtronic, AstraZeneca; Alessandra Di Lelio, Paola Del Sindaco, Francesco Reggiani, Giuseppe Lucisano: have no conflict of interest to declare.

Compliance with Ethics Guidelines. All procedures followed were in accordance with the ethical standards of the responsible 
committee on human experimentation (institutional and national) and with the Helsinki Declaration of 1964, as revised in 2013. Informed consent was obtained from all patients for being included in the study.

Open Access. This article is distributed under the terms of the Creative Commons Attribution-NonCommercial 4.0 International License (http://creativecommons.org/licenses/ by-nc/4.0/), which permits any noncommercial use, distribution, and reproduction in any medium, provided you give appropriate credit to the original author(s) and the source, provide a link to the Creative Commons license, and indicate if changes were made.

\section{REFERENCES}

1. European Medicines Agency. Insulin degludec. Summary of product characteristics; 2013. http:// www.ema.europa.eu/docs/en_GB/document_library/ EPAR_-_Product_Information/human/002498/WC50 0138940.pdf. Accessed 17 Sept 2018.

2. Garber AJ, King AB, Del Prato $S$, et al. Insulin degludec, an ultra-longacting basal insulin, versus insulin glargine in basal-bolus treatment with mealtime insulin aspart in type 2 diabetes (BEGIN basal-bolus type 2): a phase 3, randomised, openlabel, treat-to-target non-inferiority trial. Lancet. 2012;21:1498-507.

3. Zinman B, Philis-Tsimikas A, Cariou B, et al. Insulin degludec versus insulin glargine in insulin-naive patients with type 2 diabetes: a 1-year, randomized, treat-to-target trial (BEGIN Once Long). Diabetes Care. 2012;35:2464-71.

4. Gough S, Bhargava A, Jain R, Mersebach H, Rasmussen S, Bergenstal RM. Low volume insulin degludec $200 \mathrm{U} / \mathrm{ml}$ once-daily improves glycemic control similar to insulin glargine with a low risk of hypoglycemia in insulin-naive patients with type 2 diabetes: a 26-week, randomized, controlled, multinational, treat-to-target trial: the $\mathrm{BEGIN}^{\mathrm{TM}}$ LOW VOLUME trial. Diabetes Care. 2013;36:2536-42.

5. Meneghini L, Atkin SL, Gough SC, et al. The efficacy and safety of insulin degludec given in variable once-daily dosing intervals compared with insulin glargine and insulin degludec dosed at the same time daily: a 26-week, randomized, open-label, parallel group, treat-to-target trial in individuals with type 2 diabetes. Diabetes Care. 2013;36:858-64.

6. Onishi Y, Iwamoto Y, Yoo SJ, Clauson P, Tamer SC, Park S. Insulin degludec compared with insulin glargine in insulin-naive patients with type 2 diabetes: a 26-week, randomized, controlled, PanAsian, treat-to-target trial. J Diabetes Investig. 2013;4:605-12.

7. Heise T, Hermanski L, Nosek L, Feldman A, Rasmussen S, Haahr H. Insulin degludec: four times lower pharmacodynamic variability than insulin glargine under steady-state conditions in type 1 diabetes. Diabetes Obes Metab. 2014;14:859-64.

8. Heise T, Nosek L, Bottcher SG, Hastrup H, Haahr H. Ultra-long acting insulin degludec has a flat and stable glucose lowering effect in type 2 diabetes. Diabetes Obes Metab. 2012;14:944-50.

9. Cryer PE. Hypoglycaemia: the limiting factor in the glycaemic management of type 1 and type 2 diabetes. Diabetologia. 2002;45:937.

10. Wild D, von Maltzahn R, Brohan E, Christensen T, Clauson P, Gonder-Frederick L. A critical review of the literature on fear of hypoglycemia in diabetes: implications for diabetes management and patient education. Patient Educ Couns. 2007;68:10-5.

11. Rodbard HW, Gough S, Lane W, Korsholm L, Bretler DM, Handelsman Y. Reduced risk of hypoglycemia with insulin degludec versus insulin glargine in patients with type 2 diabetes requiring high doses of basal insulin: a meta-analysis of 5 randomized begin trials. Endocr Pract. 2014;20:285-92.

12. Meece J. Dispelling myths and removing barriers about insulin in type 2 diabetes. Diabetes Educ. 2006;32:9S-18S.

13. de Lusignan S, Crawford L, Munro N. Creating and using real-world evidence to answer questions about clinical effectiveness. J Innov Health Inf. 2015;22:368-73.

14. Garrison LP Jr, Neumann PJ, Erickson P, Marshall D, Mullins CD. Using real-world data for coverage and payment decisions: the ISPOR Real-World Data Task Force report. Value Health. 2017;10:326-35.

15. Siegmund T, Tentolouris N, Knudsen ST, et al. A European, multicentre, retrospective, non-interventional study (EU-TREAT) of the effectiveness of insulin degludec after switching basal insulin in a population with type 1 or type 2 diabetes. Diabetes Obes Metab. 2018;20:689-97.

16. Bohn B, Zimmermann A, Wagner C, et al. Real-life experience of patients starting insulin degludec. A 
multicenter analysis of 1064 subjects from the German/Austrian DPV registry. Diabetes Res Clin Pract. 2017;29:52-8.

17. Shimoda S, Sato M, Sekigami T, et al. A 1-year, prospective, observational study of Japanese outpatients with type 1 and type 2 diabetes switching from insulin glargine or detemir to insulin degludec in basal-bolus insulin therapy (Kumamoto Insulin Degludec Observational study). J Diabetes Investig. 2016;7:3-10.

18. Melzer Cohen C, Thorsted BL, Wolden ML, Chodick G, Karasik A. Improved glycemic control achieved by switching to insulin degludec in insulin-treated patients with type 2 diabetes in a real-world setting: a non-interventional, retrospective cohort study. Diabetes Ther. 2017;8:1047-55.

19. Sullivan SD, Bailey TS, Roussel R, et al. Clinical outcomes in real-world patients with type 2 diabetes switching from first- to second-generation basal insulin analogues: comparative effectiveness of insulin glargine 300 units $/ \mathrm{mL}$ and insulin degludec in the DELIVER D+ cohort study. Diabetes Obes Metab. 2018;20:2148-58.

20. Simioni N. Inpatient hyperglycemia management: the opportunities of a new basal insulin. Ital J Med. 2016;10:103-10. 